\title{
To cement or not to cement, that is the question in elderly!
}

\author{
O. Şahap Atik, $\mathrm{MD}^{1}$ (D), Deniz Çankaya, $\mathrm{MD}^{2}$ (D) \\ ${ }^{1}$ President, Turkish Joint Diseases Foundation, Ankara, Turkey \\ ${ }^{2}$ Department of Orthopedics and Traumatology, Gülhane Training and Research Hospital, Ankara, Turkey
}

Osteoarthritis and osteoporosis are two chronic, progressive diseases of which the prevalence increases with age, ${ }^{[1]}$ as well as hip fractures. Hip arthroplasty is a successful treatment method for end-stage osteoarthritis and hip fractures in elderly. Once the surgeon decides to apply hip arthroplasty, cementation is one of the main concerns to decide and there are a variety of fixation techniques such as cementless, hybrid, reverse hybrid, and cemented. ${ }^{[2]}$

Age is one of the determinants for the fixation technique and cementless total hip arthroplasty is usually preferred over a cemented technique in patients younger than 70 years old with hip osteoarthrosis. ${ }^{[3]}$ In older patients, osteoporosis is the main problem with the accompanying low quality of the bone, leading to the failure of osseointegration in cementless fixation of the acetabular cup and femoral stem. ${ }^{[1,4]}$ Examining the acetabular cup, the cemented technique has excellent long-term result and, therefore,

Received: May 07, 2021

Accepted: May 07, 2021

Published online: June 11, 2021

Correspondence: O. Sahap Atik, MD. Turkish Joint Diseases

Foundation, Mustafa Kemal Mah., Dumlupınar Bul., 274/2

C2 Blok, Ofis 5, 06900 Çankaya, Ankara, Türkiye.

E-mail: satikmd@gmail.com

Doi: 10.52312/jdrs.2021.57900

Citation: Atik OŞ, Çankaya D. To cement or not to cement, that is the question in elderly! Jt Dis Relat Surg 2021;32(2):277-278.

02021 All right reserved by the Turkish Joint Diseases Foundation

This is an open access article under the terms of the Creative Commons Attribution-NonCommercial License, which permits use, distribution and reproduction in any medium, provided the original work is properly cited and is not used for commercial purposes (http://creativecommons.org/licenses/by-nc/4.0/). the widespread preference for cementless fixation of the acetabulum cannot be explained by a superior survival of cementless fixation technique. ${ }^{[5,6]}$

The femoral stem is one of the two main components of total hip arthroplasty. In a recent cadaveric study, cemented stem increased the load-to-failure force by $25 \%$ compared to the cementless stem, being one of the other explanations of the higher failure rate of the cementless technique. ${ }^{[7]}$ While comparing the modern uncemented femoral stem designs and cemented stems, the latter has lower rates of periprosthetic fracture. ${ }^{[8]}$ This is another disadvantage of the cementless technique that leads to higher re-operation rates in total hip arthroplasty. ${ }^{[3,8]}$

As a result, the cementless fixation technique is associated with an increased risk of revision; that is why the cemented technique is suggested to be the gold standard in older patients with 10- to 20 -year survival rates exceeding $90 \%$ in patients 75 years and older. ${ }^{[1,4]}$ Despite the advantages of the cemented fixation technique in older patients, some drawbacks exist such as bone cement implantation syndrome-induced early postoperative mortality. ${ }^{[3,9,10]}$ Although there is a widespread-dating back-belief of higher mortality after cementation, recent data from 188,606 surgeries in the Nordic Arthroplasty Register Association database ${ }^{[9]}$ and a recent randomizedcontrolled trial comparing cementless versus cemented techniques ${ }^{[10]}$ reported similar mortality rates, as well as a study examining the mortality rates in 4,509 octogenarian patients after total hip arthroplasty. ${ }^{[4]}$ In a large-scale study including 12,491 patients who underwent hemiarthroplasty, cemented technique choice did not influence hospital 
mortality $(1.7 \%$ for uncemented fixation vs. $2.0 \%$ for cemented fixation; $\mathrm{p}=0.61$ ) and overall mortality (cumulative incidence at one year after the operation: $20.0 \%$ for uncemented fixation vs. $22.8 \%$ for cemented fixation; $\mathrm{p}=0.08$ ) in hip fracture patients. ${ }^{[1]}$

Aging of the population inevitably leads to the increase in the incidence of hip fractures, and a progressive increase in the cumulative cost of the treatment causes a heavy burden for the hospitals and social security system; that is why costs are another concern for the choices of the cemented or cementless technique. While evaluating the Medicare patients, cemented femoral fixation outperformed cementless fixation in terms of the length of hospital stay, readmission, cost of care, and reoperation. ${ }^{[12]}$ Revisions after hip replacement is another cause of the increased cost, cemented fixation should be considered for the elder total hip arthroplasty patients ${ }^{[1,4]}$ and the hemiarthroplasty treatment of displaced femoral neck fractures, ${ }^{[8,11]}$ unless contraindicated.

Why do surgeons insist to use cementless fixation for all the patients, despite the strong evidence in favor of cementation for the elder total and partial arthroplasty patients? There may have been selection bias based upon surgeon training and experience, which we are unable to control the given available database information. ${ }^{[12]}$ In a low-volume education center, one can finish a fellowship or residency program without experiencing any cemented fixation; therefore, he/she may concern about his/her sufficiency of the surgical skill in applying the cemented technique. At least 10 cases per annum need to ensure the preservation of the surgical skill in cementation to minimize revision risk. ${ }^{[6]}$ In elder groups, poor bone biology is challenging in cementless fixation, and cemented fixation is the better choice with less complication, low cost, and better clinical outcomes. Therefore, surgeons should be versatile between these two techniques in their surgical skills. Surgical training programs should be organized to provide this surgical technique versatility during the residency and fellowship education.

\section{REFERENCES}

1. Atik OŞ, Aslan A, Odluyurt M. Are fragility fractures being treated properly? Jt Dis Relat Surg 2020;31:403-4.

2. Atik OŞ. Hybrid or reverse hybrid for total hip arthroplasty? Eklem Hastalik Cerrahisi 2013;24:1.

3. Lindberg-Larsen $\mathrm{M}$, Petersen PB, Jørgensen CC, Overgaard S, Kehlet H; Lundbeck Foundation Center for Fast-track Hip and Knee Arthroplasty Collaborating Group. Postoperative 30-day complications after cemented/hybrid versus cementless total hip arthroplasty in osteoarthritis patients $>70$ years. Acta Orthop 2020;91:286-92.

4. Jämsen E, Eskelinen A, Peltola M, Mäkelä K. High early failure rate after cementless hip replacement in the octogenarian. Clin Orthop Relat Res 2014;472:2779-89.

5. Van Praet F, Mulier M. To cement or not to cement acetabular cups in total hip arthroplasty: A systematic review and re-evaluation. SICOT J 2019;5:35.

6. Hanly RJ, Whitehouse SL, Lorimer MF, de Steiger RN, Timperley AJ, Crawford RW, et al. The outcome of cemented acetabular components in total hip arthroplasty for osteoarthritis defines a proficiency threshold: Results of 22,956 cases from the Australian Orthopaedic Association National Joint Replacement Registry. J Arthroplasty 2019;34:1711-7.

7. Klasan A, Bäumlein M, Bliemel C, Putnis SE, Neri T, Schofer MD, et al. Cementing of the hip arthroplasty stem increases load-to-failure force: A cadaveric study. Acta Orthop 2019;90:445-9.

8. Song JSA, Dillman D, Wilson D, Dunbar M, Richardson G. Higher periprosthetic fracture rate associated with use of modern uncemented stems compared to cemented stems in femoral neck fractures. Hip Int 2019;29:177-83.

9. Pedersen AB, Mailhac A, Garland A, Overgaard S, Furnes $\mathrm{O}$, Lie SA, et al. Similar early mortality risk after cemented compared with cementless total hip arthroplasty for primary osteoarthritis: Data from 188,606 surgeries in the Nordic Arthroplasty Register Association database. Acta Orthop 2021;92:47-53.

10. Barenius B, Inngul C, Alagic Z, Enocson A. A randomized controlled trial of cemented versus cementless arthroplasty in patients with a displaced femoral neck fracture: A fouryear follow-up. Bone Joint J 2018;100-B:1087-93.

11. Okike K, Chan PH, Prentice HA, Paxton EW, Burri RA. Association between uncemented vs cemented hemiarthroplasty and revision surgery among patients with hip fracture. JAMA 2020;323:1077-84.

12. Oh JH, Yang WW, Moore T, Dushaj K, Cooper HJ, Hepinstall MS. Does femoral component cementation affect costs or clinical outcomes after hip arthroplasty in medicare patients? J Arthroplasty 2020;35:1489-96.e4. 